\title{
EchoGéo
}

55 | 2021

Espace et parentalité

\section{Les mères, sujet politique : ancrage local et luttes pour l'égalité}

Entretien avec Fatima Ouassak, réalisé le 22 janvier 2021 à Bagnolet

Fatima Ouassak et Félicie Roux

\section{(2) OpenEdition}

\section{Journals}

Édition électronique

URL : https://journals.openedition.org/echogeo/21154

DOI : $10.4000 /$ echogeo. 21154

ISSN : 1963-1197

Éditeur

Pôle de recherche pour l'organisation et la diffusion de l'information géographique (CNRS UMR 8586)

Référence électronique

Fatima Ouassak et Félicie Roux, "Les mères, sujet politique : ancrage local et luttes pour l'égalité », EchoGéo [En ligne], 55 | 2021, mis en ligne le 03 mai 2021, consulté le 11 août 2021. URL : http:// journals.openedition.org/echogeo/21154; DOI : https://doi.org/10.4000/echogeo.21154

Ce document a été généré automatiquement le 11 août 2021.

EchoGéo est mis à disposition selon les termes de la licence Creative Commons Attribution - Pas d'Utilisation Commerciale - Pas de Modification 4.0 International (CC BY-NC-ND) 


\section{Les mères, sujet politique : ancrage local et luttes pour l'égalité}

Entretien avec Fatima Ouassak, réalisé le 22 janvier 2021 à Bagnolet

Fatima Ouassak et Félicie Roux

\section{RÉFÉRENCE}

Ouassak F.

La puissance des mères. Pour un nouveau sujet révolutionnaire

2020. Paris, La Découverte, $272 \mathrm{p}$.

1 Fatima Ouassak est consultante en politiques publiques et co-fondatrice du Front de mères, créé en 2016 qui se définit comme "le premier syndicat de parents ", et comme « une organisation politique qui lutte contre les discriminations et les violences que subissent les enfants, à travers l'action collective des parents au sein des écoles, et un projet d'auto-organisation dans les quartiers populaires. » Elle publie en août 2020 aux éditions La Découverte un ouvrage intitulé La puissance des mères. Pour un nouveau sujet révolutionnaire. 


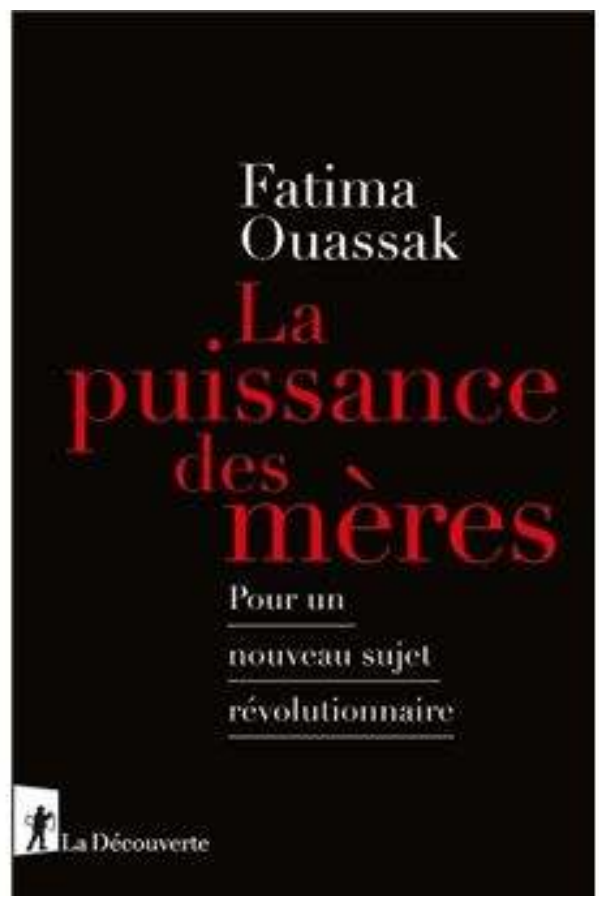

2 Cet ouvrage analyse les raisons qui ont conduit l'auteure à militer en tant que mère à Bagnolet, puis à fonder le Front de mères. A la croisée des luttes féministes, antiracistes et écologistes, elle montre en quoi les mères constituent une force politique et stratégique dans la politisation de l'enfance et de la maternité et le combat pour l'égalité. Partant de son expérience tout en s'appuyant sur une réflexion sur les rapports de domination qui structurent la société, elle resitue ces luttes dans un cadre qui mobilise différentes échelles spatiales et temporelles tout en soulignant l'importance de l'ancrage local des mobilisations.

Félicie Roux (FR). Pour commencer, je voulais revenir sur ce projet d'écriture, comment est née l'envie d'écrire ce type d'ouvrage? C'est à la fois un récit d'expériences qui comporte beaucoup d'analyse, c'est aussi un plaidoyer, peut-être un manifeste... comment le qualifiestu d'ailleurs?

Fatima Ouassak (FO). Tout d'abord, c'est important de le préciser, je suis consultante de métier, j'ai fait beaucoup de formations notamment autour des enjeux d'égalité, de lutte contre les discriminations, en direction de publics pas faciles, dans un contexte où beaucoup de ces publics, notamment les travailleurs sociaux, ont tendance à réduire la question sociale à une dimension culturaliste des choses. La grille de lecture du fait social, voire des inégalités sociales, pour beaucoup de ces travailleurs sociaux est culturaliste : ils lisent le fait social comme un fait culturel, et ils réduisent le fait culturel à des représentations qu'ils ont de la culture "africaine", " musulmane » ou « maghrébine ». L'idée est pour eux que si des personnes subissent telle discrimination, telle inégalité, c'est parce qu'elles ont telle culture, c'est à cause, en vrac, de la polygamie, de l'éducation des parents, du rapport parents-enfants, du fait qu'il n'y a pas d'affection dans ces familles-là. On retrouve cette grille de lecture culturaliste quand on parle parentalité et interculturalité. Je parle de ce contexte-là et de mon métier parce que j'ai été amenée, depuis une quinzaine d'années maintenant, à expliquer à ce type de publics la question raciale en passant par des exemples, un récit d'expériences, des exercices visant l'empathie pour amener ces 
publics, typiquement les travailleurs sociaux, les enseignants, vers une compréhension de ce qu'est la lutte contre les discriminations, les enjeux d'égalité.

Je dis ça pour expliquer comment a été construit le livre. Tu disais « comment peuxtu qualifier le livre?». En fait, il y a plusieurs parties, la partie pour moi la plus importante, c'est la dernière. C'est la partie dédiée à la stratégie politique, pour répondre à la question « qu'est-ce qu'on fait maintenant? ». Parce que tout le propos - le livre - est un projet politique, c'est une proposition politique. On est face à telle situation, je ne suis pas la première à la décrire, à l'analyser, mais j'ai une proposition à faire pour faire front, plus large que la seule organisation Front de mères. Un large front de mères - en tant que force politique et stratégique - peut participer à ce front de lutte contre les oppressions, pour le dire vite, pour mettre hors d'état de nuire le système capitaliste, avec ses dimensions raciste et patriarcale. Et alors, pour arriver à cette dernière partie, je passe par les premiers chapitres. Le premier chapitre est dédié effectivement à des récits d'expériences, notamment sur la question du racisme structurel, pour ne pas arriver avec mes gros sabots et déclarer «je vais vous expliquer ce qu'est le racisme structurel ", parce que je sais très bien, justement parce que j'ai cette expérience de formatrice et de consultante, que l'amener dans un cadre théorique peut créer des résistances ; le prétendu « racisme anti-blancs », ou le fait que je sois une femme non-blanche moi-même peut entraîner des préjugés tels que « elle va défendre sa tribu, elle est en colère "... Donc pour éviter cela, au début du livre je décris des situations en évitant de les qualifier de situations racistes, ce sont de vraies situations, mais il y a un travail d'écriture, et un travail d'analyse, ce n'est pas juste du témoignage. Je pense que ça marche plutôt bien, c'est-à-dire que les personnes qui sont concernées par la situation, qui ont la même expérience, peuvent trouver là une légitimité à dire leur vécu similaire, cela permet de sortir d'une expérience individuelle, et je pense que ça peut contribuer à faire de cette expérience une expérience politique au sens où elle est commune et peut s'expliquer par un système. Donc c'est évidemment important pour moi que les habitants des quartiers populaires, les mères des quartiers populaires, les mères racisées ou les mères tout court, selon où l'on se situe dans le livre, puissent y retrouver du vécu. Mais pour les personnes qui ne sont pas concernées, qui n'ont jamais vécu les expériences qui sont décrites, il y a aussi cette proposition à être dans l'empathie qui est implicite : « voyez ces situations, voyez plus loin dans le livre qu'elles sont confirmées par ces travaux de recherche et ces chiffres, vous ne pouvez pas nier qu'il s'agit là de racisme structurel. »

Certains parlent de "déserts politiques dans les quartiers populaires ", mais on voit bien dans le livre à quel point il est difficile de s'organiser. Ce que je montre c'est qu'à Bagnolet on a essayé de se mobiliser, et on s'est mobilisé sur les questions d'écologie. On n'avait rien, on n'avait pas de locaux, les réunions on les faisait chez nous, ça a été des conditions de lutte très difficiles pour nous. En plus on avait à dos tout le monde. Bagnolet, c'est une ville dite de gauche, encore plus hier qu'aujourd'hui, donc ce sont les communistes, mais ce sont aussi effectivement les socialistes, les écologistes... Il n'y a pas d'extrême-droite ici, vraiment parce qu'il n'y a pas besoin; l'échiquier politique national se rejoue à gauche, c'est-à-dire que la question raciale clive ici la gauche comme elle clive l'ensemble de la classe politique au niveau national. Les forces dites de gauche au niveau local n'ont de cesse que d'empêcher une population qu'elles considèrent comme devant rester sous contrôle, l'empêcher d'exister d'une 
manière ou d'une autre. Par exemple, quand nous avons voulu que nos enfants aient un repas équilibré, il n'y avait pas d'histoire de halal, on parlait juste de repas végétarien et équilibré. Pour ces forces dites de gauche, cela signifiait plus de place pour les musulmans, pour cette population essentiellement de classe populaire que l'on considère comme ne méritant pas de participer à égalité à la chose publique, à la décision politique. Je dis ça pour expliquer quelles sont nos conditions de lutte, il faut lutter pour être légitime à lutter, légitimes à simplement exister. Parce que dans le premier chapitre, j'insiste pour dire qu'avec les autres mères, nous ne voulions pas forcément mener une lutte politique, nous souhaitions juste que nos enfants aient un repas équilibré à midi. On paye des impôts comme tout le monde. Et cette simple exigence d'égalité a été disqualifiée et nous avons été stigmatisées. Quand j'allais raccompagner ma fille à l'école en petite section, des enseignantes ne me disaient plus bonjour par exemple. C'est anecdotique mais la lutte peut avoir un coût énorme au niveau local. Et justement il y a la peur, dans l'immigration, mais je pense que c'est le cas dans la classe ouvrière de manière générale, la peur des représailles, la peur pour les enfants. Mais je pense que c'est un mauvais calcul de ne pas politiser l'éducation, le foyer, notamment sur la question de racisme, et que c'est ça qui nous fait perdre encore plus. Nous gagnons beaucoup plus à politiser, à nous mobiliser, à être dans du collectif, et c'est ce que je montre dans le livre. Je suis entrée comme n'importe qui à la FCPE, je pensais vraiment que le problème à la cantine allait trouver très vite une solution d'ordre technique, le dispositif d'alternative végétarienne, et que la FCPE allait prendre en charge cette proposition. Et c'est parce que ça ne s'est pas fait - j'explique pourquoi - que l'on a donc été obligées de créer le Front de mères. Si je fais le récit et l'analyse des attaques que l'on a subies, c'est pour contrer toute l'offensive qui tend à nous qualifier de " communautaristes", dès lors qu'on se mobilise politiquement. Et je donne quelques exemples historiques où l'on voit que les membres des organisations de l'immigration et des quartiers populaires ont très souvent d'abord essayé d'agir au sein des organisations majoritaires, typiquement la CGT. Ce qui est logique, personne ne préfère militer dans sa cave, sans argent, sans réseaux. On préfère tous militer avec un minimum de moyens, avec la perspective de solutions rapides et on se dirige donc naturellement plutôt vers les organisations majoritaires. Si on se retrouve dans la cave, dans la galère, à être diabolisés, c'est parce qu'on n'a pas réussi à se faire entendre et respecter au sein des organisations majoritaires. D'ailleurs on est peu dans la cave en question parce que beaucoup arrêtent avant. Je trouve donc déplacé de parler des quartiers populaires comme de déserts politiques, où les gens ne se mobilisent pas. Avant d'apprécier le degré de mobilisation dans les quartiers populaires, on doit d'abord regarder le contexte et les mécanismes qui font que la parole politique des populations nonblanches qui vivent dans les quartiers populaires est confisquée.

FR. Oui, c'est quelque chose que j'avais noté dans ce que tu dis, à savoir la difficulté à se constituer en sujets politiques quand on est à la croisée de ces rapports de domination: être femme, musulmane, racisée, issue de classes et des quartiers populaires... Car on a d'un côté une invisibilisation de cette situation dans les organisations majoritaires, et de l'autre côté une sorte de survisibilisation qui conduit à une stigmatisation. Et donc j'ai l'impression que c'est devenu pour toi une nécessité de se saisir de ce problème-là à partir notamment de l'expérience de la parentalité dans les quartiers populaires ?

FO. Il est clair que les ennemis de la classe ouvrière et immigrée, organisations et institutions, tombent sur un os dès lors qu'il s'agit des enfants. Non pas parce qu'on est d'origine africaine, je n'essentialise pas du tout une culture africaine ou 
méditerranéenne où l'enfant serait important, la maternité et l'instinct maternel seraient plus précieux, je suis féministe et je considère donc que l'instinct maternel est une invention et une expression sexistes. Ce que je dis en revanche, c'est que les enfants sont l'une des raisons principales pour lesquelles les gens quittent leurs terres, s'exilent, et on sait à quel point la terre est importante pour nos parents africains comme pour les gens qui vivent dans le Jura, pour les Gilets jaunes. La terre, le fait de se sentir dépossédés cette terre, c'est pareil pour tout le monde. Nos parents ont quitté leurs belles montagnes pour s'entasser dans des HLM. Ma mère est arrivée en France dans les années 1970, elle a passé des mois en hôpital psychiatrique, ce n'est pas vrai de dire que nos parents sont arrivés au paradis en arrivant en France. S'ils ont quitté leur terre, souvent ils l'ont fait par sacrifice. C'est quelque chose de très important dans l'immigration, mais aussi dans la classe ouvrière, c'est pour ça que je disais que nos ennemis sont tombés sur un os quand ils oppressent nos enfants.

Si je m'organise politiquement, si on crée le Front de mères à quelques-unes, c'est pour les enfants, c'est contre les oppressions que subissent les enfants, d'abord. Après, je parle dans le livre des violences obstétricales que subissent les mères, des violences que j'ai pu subir moi-même en tant que mère. Mais si je décide de m'organiser politiquement, en tant que mère, ce n'est pas pour lutter uniquement contre les oppressions que subissent les mères, c'est d'abord pour lutter contre les oppressions que subissent les enfants. Je considère que dès lors qu'on touche à leurs droits politiques, à leurs libertés fondamentales, dès lors qu'on justifie cette confiscation au nom de la protection qu'on leur apporte en tant qu'adultes, au nom donc de cette responsabilité que l'on prend, il faut réinvestir cette idée de protection des enfants. Et notamment quand il s'agit d'enfants qui subissent particulièrement des violences sociales, particulièrement des inégalités, qui sont réduits dans leur champ des possibles, comme je l'explique dans le livre, c'est-à-dire ramenés petit à petit, par mille manières depuis la naissance jusqu'au marché du travail vers des secteurs d'activité où ils sont exploités, moins bien payés... Et du coup je renvoie à une responsabilité collective des parents en matière de protection des enfants, une responsabilité collective des mères en particulier sur certains sujets, par exemple la question des violences sexuelles, dans une perspective féministe.

FR. Et à cet égard, tu expliques que les enfants et les mères sont un peu un angle mort du féminisme, et en même temps tu proposes d'utiliser l'outil féministe pour politiser la question de l'enfance et de l'expérience de la maternité... Et il s'agit en particulier de politiser la question des violences policières, des violences sociales ainsi que des violences sexuelles, donc ce serait intégrer une sorte de continuum des violences que peuvent subir les enfants en allant de l'espace domestique à l'espace public...

FO. Absolument. Malgré la critique du féminisme à laquelle je participe, je considère que le féminisme reste un outil de libération. Je pars de ce postulat de départ. J'ai essayé dans le livre de ne pas m'enfermer, me piéger sur les biais, les manques, les lacunes, les angles morts du féminisme tels qu'il peut être qualifié, «féminisme de classe ", « féminisme blanc » même si j'en parle un peu pour expliquer pourquoi le féminisme majoritaire y compris dans sa dimension radicale ne prend pas en considération les mères. Il y a là pour moi quelque chose à travailler, un mépris de classe, considérer que les mères, c'est un truc d'arabes, d'immigrés, il y a quelque chose là qui est structurel dans le féminisme français. Et en même temps, je ne fais pas du tout le deuil du féminisme. Je ne fais le deuil d'aucun outil qui pourrait nous libérer en réalité. C'est comme l'écologie. Pourtant ceux qui m'ont le plus attaquée, ce 
sont les écologistes locaux. Et pourtant je n'en fais pas le deuil car l'écologie ne se réduit pas à cette parole écologiste-là. La question est stratégique: dans quelle mesure le fait d'investir les questions féministes nous permettrait de gagner en puissance en tant que mères par rapport aux institutions?

Reste que l'outil de libération que je considère être potentiellement le plus puissant et le plus efficace pour la classe populaire et en particulier, pour cette classe populaire qui vit dans ces territoires-là, ces quartiers populaires, c'est l'écologie. Bien loin devant le féminisme, l'antiracisme, le communisme. Évidemment une écologie du point de vue des populations qui vivent dans les quartiers populaires, une écologie dont je commence une définition dans mon livre avec au centre, la liberté de circuler dans l'espace public. Si je devais en donner une définition située, c'est comment nos enfants circulent dans l'espace public, et comment nous parents nous battons, permettons que nos enfants puissent circuler, courir, jouer dans l'espace public. Pour moi la base de l'écologie, c'est respirer. Dans les quartiers populaires, ça ne sert à rien de venir nous parler de nucléaire ou de réchauffement climatique, parce que l'urgence c'est que nos enfants sont assignés et qu'ils ne peuvent pas jouer dehors. Quand on est passés devant chez moi, je t'ai dit que là les parents ne laissent pas leurs enfants jouer dehors parce qu'on vit au-dessus d'un commissariat, et surtout parce qu'il y a du trafic dans le quartier. Les enfants des quartiers populaires ne peuvent pas jouer dehors, alors que pour d'autres ce n'est pas une question, ce n'est pas un enjeu. En France, la liberté de circuler est évidente pour plein de gens, ils peuvent circuler sans être entravés dans l'espace public. D'ailleurs, on peut poser aussi la question d'un point de vue féministe : pourquoi les femmes ne peuvent pas circuler librement dans l'espace public la nuit ? Il n'y a personne qui leur dit «c'est interdit de circuler la nuit ». Mais évidemment il y a des formes là aussi d'assignation à résidence, on se dit qu'on prend des risques à sortir, et puis il y a tout l'imaginaire autour du viol.

C'est une définition un peu longue, parce qu'elle est un bricolée, elle est très située ma définition de l'écologie, elle n'est pas du tout majoritaire, elle prend vraiment en compte cette question de l'assignation à résidence, l'histoire de l'immigration et de la classe ouvrière, le fait qu'on soit totalement dépossédés de pouvoir politique. C'est vraiment avec le Front de mères que je me suis rendu compte à quel point nous étions dépossédés. Quand les écologistes militent comme dans le $2^{\text {ème }}$ arrondissement de Paris, pour l'alternative végétarienne, ils l'obtiennent. En deux minutes. C'est un problème technique : le choix entre un plat sans viande et un plat avec viande. Quand ce sont des écologistes "classiques", ils l'ont. Mais quand ce sont des femmes musulmanes comme nous, ça prend cinq ans. Donc la question de l'écologie pose la question du pouvoir politique : quel pouvoir a-t-on? Et aussi comment reprendre du pouvoir politique, reprendre ce qu'on nous a confisqué?

FR. Et justement, sur cette question du territoire, tu proposes une analyse assez relationnelle et dynamique qui mobilise différentes échelles spatiales et temporelles qui te semblent pertinentes pour expliquer dans quel cadre spatio-temporel s'inscrivent ces luttes de mères: ce sont des mobilisations qui ont commencé avec la question écologique à l'échelle locale, qui se situent à l'échelle nationale pour créer un rapport de force politique avec le syndicat Front de mères et tu fais beaucoup référence à la question de l'histoire 
migratoire, ce qui renvoie à une échelle transnationale de la trajectoire familiale. Tu inscris donc aussi ces luttes dans un temps long...

FO. Oui. Absolument. Mais aussi pour visibiliser, alors même pas des populations, mais plutôt des luttes, les luttes que mènent ces populations dans un pays, la France, qui a tendance à dire que ces luttes n'ont pas existé. C'est-à-dire que ce qui est mobilisé en France, concernant cette population originaire d'Afrique, de pays anciennement colonisés, ce sont des représentations autour de leur passivité, de leur docilité, encore plus aujourd'hui qu'hier. Alors qu'on sait très bien qu'il y a des luttes qui ont été menées. Par exemple à propos de Mai 68, tu vois des photos des ouvriers de l'époque qui faisaient grève, c'est beaucoup de moustachus du pays, nos pères. On a une représentation de l'immigré - lire le travail du sociologue Abdelmalek Sayad passif, docile, qui n'a pas de mémoire, qui n'a pas d'histoire. Il y a donc cette nécessité de dire : des luttes ont été menées, et nous, enfants de ces gens-là sommes héritiers de ces luttes-là aussi. Ces luttes éduquent nos enfants qui voient bien que, leurs mères, ce ne sont pas les mères soumises qu'on leur décrit à longueur de temps, à l'occasion du 8 mars il y a quelques années par exemple avec cette idée que "c'est pire chez les Arabes que chez nous ". L'égalité femmes/hommes, ça va à peu près en France, mais par contre là-bas, c'est la polygamie, l'excision, des choses horribles. Le patriarcat paraît toujours plus horrible chez le voisin, surtout quand il est non-blanc. Le fait de dire aux enfants que leurs mères, leurs grand-mères avaient mené des luttes, et même fait la guerre, libéré leurs pays de la colonisation, c'est pour qu'ils soient fiers de leurs héritages, de leurs ancêtres. C'est la dernière partie du livre qui est consacrée à la transmission d'une capacité à ne pas se résigner, au-delà des identités. La question des identités ne m'intéresse pas vraiment, ce n'est pas mon sujet. Ce qui m'importe c'est la capacité à ne pas se résigner. Et c'est pour ça que ces luttes sont importantes. Ça c'est la première chose.

Et deuxièmement, sur l'histoire de laisser une trace, d'en dire quelque chose, de visibiliser ces luttes, c'est aussi pour dire que ce n'est pas que pour les Noirs et les Arabes, c'est pour tout le monde. Car soit on invisibilise ces luttes, soit quand on les visibilise, c'est pour dire « regardez ce qui se passe dans les quartiers. » Et c'est les quartiers pour les quartiers. On serait une sous-catégorie de la question sociale. C'est pour cela que j'insiste bien dès le préambule du livre sur le fait qu'il s'agit d'un point de vue situé, mais qu'il s'agit d'un projet universel. C'est pour tout le monde. Oui parce que si le livre avait été écrit par une femme blanche qui a un projet politique pour tout le monde, et qui pourtant elle aussi est située - habitant par exemple dans le $16^{\text {ème }}$ ou à Montreuil, être de classe moyenne supérieure - on n'aurait pas dit « attention c'est une proposition politique pour les classes moyennes supérieures des quartiers pavillonnaires. " Lorsqu'une femme blanche CSP + parle, là elle n'est pas sous-catégorisée, on considère qu'elle parle pour tout le monde, y compris pour moi. C'est pour ça que je parle des Sonacos ou des Folles de la place Vendôme. Les Sonacos ont véritablement créé et lancé la question du Droit au logement qui concerne tout le monde aujourd'hui. Sans que personne ne le reconnaisse. Ça paraît complètement aberrant de dire qu'une lutte avec une dimension universelle pourrait venir d'un groupe minoritaire. Or, c'est le cas. Et pour les Folles de la place Vendôme, j'ai cité leurs propos exprès : elles appelaient bien les mères du monde entier à les rejoindre. 
FR. Et d'ailleurs tu parles aussi de l'alliance avec les mères des Gilets jaunes que tu as rencontrées...

FO. Absolument. D'ailleurs, à y regarder de plus près, je pense que personne ne veut autant que nous militants des quartiers populaires travailler avec tout le monde, parce qu'à un moment donné, il faut amener tout le monde sur des valeurs, des principes, et un projet politique...

FR. ... qui est la cause pour l'égalité globalement... ?

FO. Oui, et c'est un projet commun. Alors je sais que c'est compliqué... dans le livre, j'amène partie après partie vers ce qui ressemble effectivement à un manifeste à la fin du livre. Je sais que c'est compliqué les rapports sociaux de domination ; j'en parle et je ne vais pas le nier. De toute façon, selon moi, réussir, c'est passer par cette étape lucide par rapport au système tel qu'il fonctionne. Mais malgré ce système - ces rapports sociaux de domination - je crois en un projet politique commun. Ce n'est pas au-delà de notre couleur, de notre sexe, de notre classe sociale, parce que ce n'est pas vrai, il y a des rapports sociaux de domination, mais ce qui compte, c'est comment on travaille ensemble au projet politique qu'on défend.

FR. Et c'est pour ça que tu parles beaucoup de l'importance de l'ancrage, de partir du concret, du quotidien...

FO. Absolument. C'est ce qui paye, et selon moi le territoire, c'est un autre sujet politique fondamental du livre. Parce que je vois bien que les organisations antiracistes en France, mais féministes aussi et un peu écologistes, majoritaires on va dire, sont complètement désancrées justement, hors sol, et le résultat, c'est qu'on a des idéologies, des projets politiques de classe. Pourquoi le féminisme français peut-il se permettre de ne pas parler des mères comme sujet politique? Quand tu n'es pas ancré dans le territoire, et dans la classe populaire, tu passes à côté de tout. Tu passes à côté de la question sociale.

FR. Et tu disais plus tôt que parfois les mouvements antiracistes, féministes ou écologistes restaient dans un ancrage très théorique, et que ce n'était pas toujours facile, d'un point de vue stratégique notamment, de mobiliser là-dessus dans une perspective de politisation... ?

FO. Absolument. En Argentine, elles viennent d'obtenir le droit à l'IVG, et en revoyant les manif de 2018, on voit les masses de milliers de femmes, beaucoup sont de la classe populaire, et elles avaient toutes le même discours féministe, et c'était populaire. Mais en France, nous féministes, dès lors qu'on organise une conférence à trente à Paris, dans une belle salle, on est contentes parce qu'on va avancer dans la réflexion, l'intersectionnalité, l'inclusivité, etc. C'est comment les trente personnes vont se performer sur un discours, le plus pertinent, le plus juste intellectuellement... Et c'est toute la tradition intellectuelle française. Les mêmes considèrent que la maternité, c'est aliénant. Il n'y a pas cet effort pour qu'on soit les plus nombreuses possibles. Comment on fait pour que les femmes de la campagne française se sentent concernées par ce qu'on dit ? Il n'y a pas cet effort-là qui est fait. En Espagne ou en Italie, il y a cet effort-là de construire un féminisme populaire. Pas du tout en France. Et pour moi la manière d'être dans des vraies luttes et qui vont marcher, qui vont nous conduire à la victoire, c'est d'être dans le territoire.

Et c'est aussi, j'en parle aussi, parce qu'on est attaqués par le territoire. Il n'y a pas en France des lois qui te disent " on va discriminer les Noirs et les Arabes, on va discriminer les enfants, on va discriminer les femmes ». Ça n'existe pas. Il y a une égalité des droits en France. Par contre, il y a une inégalité de traitement, à travers 
les politiques publiques, et je suis consultante en politiques publiques, donc je vois très bien comment ça passe dans la tuyauterie...

\section{FR : Elle passe par une territorialisation...}

FO : J'avais travaillé sur, j'en parle un peu dans le livre, une évaluation de tous les projets de lutte contre les violences intrafamiliales dans les années 2000 et quelques, après 2005. Et ça a duré plusieurs années. Les commanditaires étaient le Ministère de la Justice et le secrétariat des droits des femmes, et puis la Politique de la ville, à l'époque ce n'était pas encore le CGET [Commissariat général à l'égalité des territoires], c'était le FASILD [Fonds d'aide et de soutien à l'intégration et à la lutte contre les discriminations]. Et à l'époque tout de suite on nous a posé l'étude ENVEFF [Enquête sur les violences envers les femmes en France], qui date de 2001, et qui montre que les violences intrafamiliales, notamment conjugales, les viols, etc., c'est dans tous les milieux, quelle que soit la classe sociale. Et les institutions étaient bien obligées de considérer que viols, violences, c'était dans tous les milieux sociaux. Il y avait déjà après 2001 cette idée que tout le monde n'était pas concerné de la même manière. Mais ce discours était surtout présent dans les médias, il y avait quand même cette étude-là [l'enquête ENVEFF] dans l'institution. Mais dès lors qu'on a eu le programme à évaluer, je me suis rendu compte avec cette évaluation que les actions étaient financées au titre de la Politique de la ville et en particulier du fonds de prévention de la délinquance. Quels sont les territoires visés par la Politique de la ville? Et en plus par le fonds de prévention de la délinquance? Ce ne sont pas les

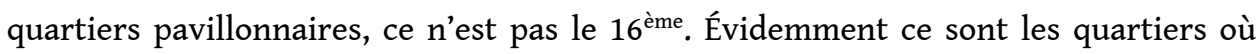
vivent les populations descendantes de l'immigration postcoloniale. Tous les espaces populaires n'étaient pas visés, pas les campagnes, ce sont vraiment ces quartiers-là. Par conséquent, on a au départ une question qui concerne tout le monde officiellement, même dans la littérature institutionnelle, et grâce à la tuyauterie institutionnelle et notamment à travers les financements des actions, on arrive à faire que cette question-là ne concerne finalement que les Noirs et les Arabes, via les quartiers Politique de la Ville. Voilà comment tu crées de l'inégalité, de la discrimination, de la stigmatisation via le territoire, une territorialisation.

C'est un vrai débat d'ailleurs dans l'antiracisme le territoire, hors sol/pas hors sol, la question de l'ancrage dans les territoires.... Moi je suis parfois qualifiée de «terteriste » par exemple. Mais j'assume cette importance accordée au territoire, à la terre. Au Front de mères, on ne peut pas rentrer si on n'est pas rattaché quelque part localement. Parce que c'est trop facile, t'arrives on ne sait pas où tu travailles, où tu vis. Toi tu protèges ta vie privée, mais par contre tu viens avec un discours antiraciste ultra radical. Mais le problème, c'est que les gens qui eux ont leurs enfants qui fréquentent telle école, qui ont leurs noms, leurs prénoms, qui travaillent à un endroit, etc. tu leur fais porter les risques que toi tu refuses de prendre. Soit dit en passant, je comprends parfaitement qu'on veuille se protéger et protéger ses proches en ne dévoilant pas son nom, la ville où l'on vit, l'endroit où on travaille ou l'université où on étudie. Mais on ne peut pas, dans ce cas, pousser à la radicalité les gens qui vivent et militent dans les quartiers populaires de manière non-anonyme.

Donc celles qui décident de la stratégie, elles sont quelque part, c'est elles qui ont priorité. Parce que tout est à doser. Tout à l'heure, je t'ai dit ce qui nous est arrivé après l'attentat dont a été victime Samuel Paty, ça peut vraiment couler notre organisation. Ça peut détruire notre vie, tout peut dégringoler, alors même que 
jusqu'alors tout allait bien. Le risque est trop grand, donc la stratégie s'expérimente et se décide dans les territoires.

FR: Dans les territoires tout en ayant ce souhait de construire un rapport de force politique au niveau national. Et c'est un combat contre les institutions, tu dis dans ton livre « l'école, c'est la guerre ", et en même temps, un combat avec les institutions en un sens parce que tu évoques une alliance avec des acteurs institutionnels, et une volonté de conquérir le pouvoir politique local par les urnes...

FO : Oui parce qu'ici en Seine-Saint-Denis, de manière générale, il y a des gens qui parlent pour nous, des élus locaux qui ne sont pas d'ici, qui ne connaissent rien des réalités du territoire, et qui se soucient peu des enjeux de la population qui y vit. Or le 93 est très attaqué médiatiquement, politiquement. Surtout parce que beaucoup des luttes sociales, des luttes de l'immigration partent de la Seine-Saint-Denis, il y a ici beaucoup de résistances, de fait parce que beaucoup de populations immigrées ont été concentrées ici. Avec tout ce qu'il y a de luttes, de résistance, a-t-on besoin de gens de gauche parachutés depuis je ne sais où? Non.

Le pouvoir politique en France, aujourd'hui, est concentré dans le système électoral. Il n'y a pas de véritables espaces d'autonomie politique, ou si peu. A partir du moment où le pouvoir est dans les élections, il faut aller le prendre.

Prendre le pouvoir, ça ne veut pas dire en faire quelque chose de tyrannique. C'est un levier de transformation sociale.

Et il ne faut pas croire que cet État va protéger nos enfants... L'État produit des inégalités, produit des discriminations qu'elles soient sexistes, racistes, justement territoriales. C'est pour cela qu'il faut un féminisme offensif : l'État ne protège pas? Alors on va le faire nous-mêmes, on va protéger nos enfants nous-mêmes. Donc il y a ça, il y a effectivement assumer d'avoir un rapport de force politique avec l'institution et donc ça veut dire avoir un pied dans l'institution, et moi j'assume complètement d'avoir un pied dans l'institution. Je suis consultante, je travaille avec les pouvoirs publics. Et en même temps, c'est essayer de concilier ça avec de l'autoorganisation, ce n'est pas incompatible. Et le Front de mères, c'est vraiment cette expérimentation-là : un pied dans le quartier, un pied dans l'école. On a un discours radical sur le système discriminatoire, le système inégalitaire, ça ne nous empêche pas de travailler avec l'institution, on cherche le rapport de force politique le plus favorable. Le but étant que nos enfants grandissent heureux et respectés dans leur dignité.

\section{AUTEURS}

\section{FATIMA OUASSAK}

Fatima Ouassak est consultante en politiques publiques et co-fondatrice du Front de mères. 


\section{FÉLICIE ROUX}

Félicie Roux est doctorante et ATER à l'Université Paris-Est Marne-la-Vallée, et membre de Lab'Urba. 